\title{
GESTÃO DE SUPRIMENTOS DE UMA UNIDADE DE TERAPIA INTENSIVA: PERCEPÇÃO DOS PROFISSIONAIS DE SAÚDE SOBRE A OCORRÊNCIA DE INFECÇÕES HOSPITALARES ASSOCIADAS ÀS FALHAS DE ABASTECIMENTO
}

\section{SUPPLY MANAGEMENT OF AN INTENSIVE CARE UNIT: PERCEPTION OF HEALTH PROFESSIONALS ABOUT THE OCCURRENCE OF NOSOCOMIAL INFECTIONS RELATED TO SUPPLY FAILURE}

\section{D0I: http://dx.doi.org/10.21714/raunp.v8i2.1242}

\section{Walquiria Barcelos de Figueiredo}

Enfermeira chefe da UTI Clínica Médica do Pronto Socorro do Instituto Central do HCFMUSP. Possui Mestrado Profissional em Gestão de Sistemas de Saúde pela Universidade Nove de Julho - UNINOVE. E-mail: walquiria.figueiredo@hc.fm.usp.br

\section{Simone Aquino}

Doutora. Professora do Programa de Mestrado Profissional em Administração - Gestão em Sistemas de Saúde da Universidade Nove de Julho - UNINOVE. E-mail: siaq06@hotmail.com

\section{Marcos Roberto Piscopo}

Doutor. Professor Programa de Pós-Graduação em Administração (PPGA) e do Programa de Mestrado Profissional em Administração - Gestão de Projetos (PMPA - GP) da UNINOVE. E-mail: piscopomr@gmail.com

Envio em: Julho de 2015

Aceite em: Junho de 2016

\section{RESUMO}

0 gerenciamento de suprimentos hospitalares em uma Unidade de Terapia Intensiva (UTI) deve suprir às boas condições durante 0 trabalho. 0 presente estudo objetivou compreender a percepção de profissionais da área de saúde de um hospital público terciário, identificando os riscos associados à falta de materiais essenciais na assistência ao paciente e ocorrência de infecções hospitalares ou infecções relacionadas à saúde. Um questionário semiestruturado foi elaborado para a equipe da unidade estudada, composta por médicos, enfermeiros, técnicos de enfermagem, auxiliares de enfermagem e fisioterapeutas. Foi realizado um levantamento e análise documental dos casos registrados de infecções relacionadas à saúde na unidade entre 2012 e 2013, análise das informações contidas nas planilhas de fornecimento de medicamentos, insumos e equipamentos de proteção individual. 0 s resultados demonstraram que $69 \%$ dos profissionais associam o risco de infecções hospitalares à falta de aventais descartáveis e luvas em uma UTI, gerando sofrimento e insatisfação no desempenho da assistência ao paciente. A gestão de compras e abastecimento de insumos para a UTI deve ser um foco de controle das Comissões de Controle de Infecção Hospitalar para criar estratégias na prevenção da ocorrência de eventos adversos, aumentar a satisfação dos profissionais e garantir a biossegurança dos pacientes e colaboradores.

Palavras-chave: UTI. IRAS. Gestão em Saúde. Sofrimento. Falha no Abastecimento. 


\section{ABSTRACT}

The management of supplies in a hospital intensive care unit (ICU) must meet the good conditions at work. This study aimed to understand the perception of professionals from the health area of a tertiary public hospital, identifying the risks associated with lack of essential materials in patient care and nosocomial infections or health-related infections. A semi-structured questionnaire was designed to study the unit team, consisting of doctors, nurses, practical nurses, nursing assistants and physical therapists. A survey and document analysis of reported cases of health-related infections in the unit between 2012 and 2013, analysis of the information contained in spreadsheets supply of medicines, supplies and personal protective equipment was conducted. The results showed that $69 \%$ of professionals associate the risk of hospital infections to lack of disposable aprons and gloves in an ICU, causing suffering and dissatisfaction in the performance of patient care. The procurement management and supply of inputs to the ICU should be a control focus of Infection Control Committees to develop strategies to prevent the occurrence of adverse events, increase the satisfaction of professionals and ensure the biosecurity of patients and employees.

Key Words: UTI. IRAS. Gestão em saúde. Sofrimento. Falha no abastecimento.

\section{INTRODUÇÃO}

O Brasil ainda enfrenta uma realidade adversa daquilo que se pode julgar satisfatório: carência de recursos humanos e materiais nas instituições de saúde (principalmente nas públicas), ausência de Comissões de Controle de Infecção Hospitalar (CCIHs) atuantes em grande parte dos hospitais, ou ainda profissionais que exercem a função sem conhecimento adequado da atividade - o que resulta em elevadas taxas de infecção hospitalar, ocorrência de surtos não detectados em berçários e unidades de terapia intensiva, emergência de bactérias resistentes a diversos antibióticos e elevado risco ocupacional (BATISTA, 2004).

Segundo Figueiredo e Zuffi (2012), além do conhecimento técnico científico, é preciso que os profissionais tenham boas condições de trabalho para prestar uma assistência satisfatória. De acordo com Garcia et al. (2013) a oferta de produtos com qualidade para higienização das mãos, além de promover segurança para o profissional e para o paciente, pode ser traduzida por satisfação e incentivo ao seu comprometimento.

Materiais são considerados insumos ou fatores produtivos, de natureza física, com determinada durabilidade, empregados na realização de procedimentos/atividades assistenciais aos pacientes. A escassez de alguns destes, considerados imprescindíveis para assistência é um dos pontos que mais afligem os gestores dos serviços de enfermagem (MESQUITA et al., 2005).

Administrar os recursos materiais com o mínimo de recursos alocados em estoques é evitar a falta de materiais médico-hospitalares e medicamentos são, sem dúvida, a principal missão do gestor de materiais hospitalares. Segundo Lanna (2010) a falta de materiais tem muitos motivos, tais como: falta de recursos financeiros; falta de atenção gerencial; falta de treinamento do pessoal da farmácia; falta de treinamento do pessoal do almoxarifado; falta de cultura de planejamento/programação por parte dos usuários que solicitam materiais de última hora; falha ocasional de fornecedores; dificuldades logísticas como alagamentos e queda de barreiras; ausência de estoques de segurança no hospital exatamente para prevenir essas faltas.

Vecina e Reinhardt (1998) também já consideraram as possíveis falhas no abastecimento de materiais para uso na assistência à saúde. A falta de insumos no almoxarifado dos serviços força os profissionais de saúde a quebrar protocolos (padronização de um processo para uniformizar uma ação) e técnicas, muitas vezes improvisadas por materiais similares aos ideais, para realização dos procedimentos, o que por sua vez acarretam riscos de falhas/eventos adversos ao paciente, bem como possível elevação de custos devido ao tratamento destes efeitos gerados. Os mesmos autores ainda agruparam as falhas em três causas: causas estruturais; causas organizacionais e causas individuais.

Nas instituições de saúde, especialmente nos hospitais, a Gestão de Recursos Materiais (GRM) constitui-se em uma questão particularmente importante, considerando a diversidade de materiais utilizados, seu elevado custo, especialmente daqueles ligados à assistência das pessoas hospitalizadas. A administração destes, normalmente, é realizada pela área administrativa, sem o envolvimento devido das áreas técnicas (HONÓRIO; ALBUQUERQUE, 2005) e, devido a isso, torna-se importante que profissionais das CCIHs estejam envolvidos na seleção de materiais diretamente relacionados na assistência ao paciente, 
pois critérios rigorosos e baseados em pesquisas devem direcionar a escolha destes materiais. Conforme a Portaria n 2616 publicada pelo Ministério da Saúde (MS, 1998), cabe à CCIH a adequação, implementação e supervisão das normas e rotinas técnico-operacionais, visando a prevenção e controle das infecções hospitalares.

Outro item fundamental na assistência ao paciente em ambiente hospitalar se refere aos Equipamentos de Proteção Individual (EPIs). O EPI é um material usado como barreira mecânica entre a transmissão materiais biológicos potencialmente contaminados (sangue, secreções, etc.) e o profissional da saúde (BATISTA, 2004) e, portanto, o não uso do EPI poderá favorecer a contaminação do profissional e permitir a transmissão de infecção hospitalar ou infecções relacionadas à saúde (IRAS).

Os itens que compõem os EPIs (luva, máscara, avental, óculos, etc.) devem estar disponíveis ao profissional de saúde durante todo o tempo de trabalho e diversos insumos (papel toalha, gaze, sabão, antissépticos, etc.) são imprescindíveis no cuidado e prevenção das IRAS. Além de causar estresse na equipe multiprofissional, a falta de um material de consumo nas unidades hospitalares leva à descontinuidade da assistência prestada e consequentemente danos ao paciente (MENDES; CASTILHO, 2009).

Na UTI estudada observa-se que o abastecimento de alguns insumos (luvas, sabão líquido e preparações alcoólicas, por exemplo) não ocorre de forma sistemática. Além disso, não é permitido estoques de materiais em armários no interior da UTI, sendo o abastecimento em fluxo constante advindo de um almoxarifado central.

As falhas no fluxo de abastecimento de materiais essenciais para a assistência ao paciente são constantes na unidade estudada, porém nunca foram mensuradas e analisadas em função da percepção do colaborador (médicos, enfermeiros, fisioterapeutas, auxiliares de enfermagem e técnicos de enfermagem) sobre a incidência de IRAS.

A culpa e sofrimento dos profissionais pela falha na segurança do paciente e medo dos riscos de contaminação por não possuir materiais básicos e EPIs foi o ponto de partida para este estudo de caso, no período de 2012 a 2013. A questão de pesquisa, portanto, a ser elucidada é: Como os profissionais atuantes em uma UTI pública reagem frente às falhas de abastecimento de insumos essenciais e EPIs, empregados na assistência ao paciente, durante a ocorrência de IRAS?
O trabalho está estruturado em mais cinco seções, além da introdução apresentada que contextualiza o tema de pesquisa, objetivos e a estrutura do trabalho. A revisão teórica aborda os principais pilares teóricos relacionados ao estudo e, em seguida, são apresentados os procedimentos metodológicos utilizados para o desenvolvimento da pesquisa a fim de subsidiar dados às proposições do estudo. Na seção de resultados e discussão são apresentados os achados da pesquisa com a análise sob a óptica da literatura pertinente e, finalmente em conclusões, também são apontadas as limitações do estudo e as recomendações futuras para a prática profissional.

\section{REVISÃO DA LITERATURA}

\subsection{GESTÃO DE SUPRIMENTOS HOSPITALARES E IRAS}

O compromisso dos profissionais da equipe de saúde no gerenciamento de recursos que viabilizam o processo de cuidar é indispensável para uma assistência isenta de riscos; e o uso racional dos materiais e equipamentos, indispensáveis ao desenvolvimento das atividades envolvidas neste processo (MADUREIRA et al., 2000).

De acordo com Vecina e Reinhardt (1998), o objetivo básico da administração de materiais consiste em colocar os recursos necessários ao processo produtivo com qualidade, em quantidades adequadas, no tempo correto e com o menor custo. O estoque é um investimento que envolve o comprometimento de recursos que poderiam ser aplicados em outras atividades e, segundo Brandalise, Pertile e Bortoli (2009), tem de estar pronto para qualquer tipo de atendimento. Porém, sua existência é indispensável ao funcionamento de um hospital. Para o funcionamento do estoque é importante ter dados históricos de consumo de cada item, para projeções de demanda dentro de níveis adequados, que permitam suprir as necessidades de consumo com estoques menores (PASCHOAL; CASTILHO, 2010).

Busca-se a otimização organizacional que reduza as necessidades de estoques. No limite, a situação ideal é a do estoque zero (PAULUS, 2005). Nos momentos de emergência não podem ocorrer faltas de produtos; além disso, o que sobra no estoque não pode ser simplesmente descartado. Portanto, a previsão de demanda deve ser bem feita, bem como a ga- 
rantia da qualidade oferecida por seus fornecedores (BRANDALISE et al., 2009).

Visando aprimorar a gestão dos riscos associados ao trabalho dos profissionais lotados na UTI, é importante a adesão a medidas preventivas durante a realização da assistência a pacientes, como as precauções padrão. No estudo de Pereira et al. (2013) os autores empregaram a Escala de Disponibilidade de EPI, onde o escore médio foi de 4,31 (DP $=0,69)$, ou seja, embora as respostas tenham sido positivas, foi demonstrado a falta de EPIs (inacessíveis nas unidades).

Oferecer equipamentos e materiais de consumo nas condições e em número adequados atende a uma premissa básica solicitada no processo de acreditação, que é estrutura básica (recursos), capaz de garantir assistência para a execução coerente de suas tarefas. Cumprir as normas de biossegurança com EPIs adequados em todo o tempo de trabalho é a garantia para o profissional de saúde. A Norma Regulamentadora 32 (NR 32), sobre segurança do trabalho, define como Risco Biológico a probabilidade da exposição ocupacional a agentes biológicos (microrganismos, parasitas, toxinas e os príons). Atualmente existe uma mobilização nos hospitais para atender às solicitações impostas pela NR 32 (CAMPOS; DAVID, 2011).

A cada ano ocorre nos EUA entre 5 a 10 episódios de pneumonia relacionada a assistência à saúde por 1.000 admissões. Segundo a Agência Nacional de Vigilância Sanitária (ANVISA, 2013) estas infecções são responsáveis por $15 \%$ das IRAS e aproximadamente $25 \%$ de todas as infecções adquiridas nas UTIs.

A Infecção Primária de Corrente Sanguínea (IPCS) está entre as mais comumente relacionadas à assistência à saúde. A IPCS associa-se a importante excesso de mortalidade, a maior tempo de internação e a incrementos de custos relacionados à assistência. A mortalidade varia entre pacientes (a mortalidade por IPCS pode atingir até $69 \%$ ). Com as ações de pacotes de medidas preventivas os chamados Bundles, houve uma redução da IPCS de modo consistente e duradouro. Este pacote compreende medidas como higiene das mãos; uso de EPIs (gorros, máscaras, avental e luvas estéreis); uso de soluções antissépticas, etc. (ANVISA, 2013). Outras medidas específicas para a prevenção e o controle da disseminação/propagação de agentes infecciosos são recomendadas por diferentes fontes, conforme demonstrado no Quadro 1.

QUADRO 1. FONTES DE RECOMENDAÇÕES PARA USO DE INSUMOS E EPIS EM AMBIENTE HOSPITALAR.

\begin{tabular}{|l|l|}
\hline ANVISA, 2013 & $\begin{array}{l}\text { Enfatiza a importância da higienização das mãos para todos os } \\
\text { profissionais de saúde. }\end{array}$ \\
\hline \multirow{2}{*}{ RDC n 42 ANVISA, 2010 } & $\begin{array}{l}\text { Disponibilizar continuamente insumos para a correta higienização } \\
\text { das mãos. }\end{array}$ \\
\hline \multirow{2}{*}{ Nota Técnica $n^{\circ} 01$ ANVISA/2013 } & $\begin{array}{l}\text { Disponibilizar continuamente EPIs para o manejo de pacientes e } \\
\text { suas secreções, além da correta paramentação para lidar com o } \\
\text { ambiente em torno do paciente. }\end{array}$ \\
\cline { 2 - 2 } & $\begin{array}{l}\text { Aplicar as medidas de precaução de contato, para os profissionais } \\
\text { que entram em contato direto com o paciente, incluindo o reforço } \\
\text { nas medidas de higiene do ambiente. }\end{array}$ \\
\hline
\end{tabular}

Fonte: Elaborado pelos autores.

Portanto, para a realização de um trabalho seguro dentro das normas de biossegurança, o abastecimento de insumos e EPIs são fundamentais como medidas preventivas de infecção hospitalar ou IRAS É fundamental conhecer a percepção dos profissionais de saúde em frente às falhas no abastecimento de materiais, suas consequências e/ou não conformidades na assistência ao paciente, a fim de que as CCIHs possam intervir devidamente na gestão de suprimentos hospitalares.

\subsection{ESTRESSE E SOFRIMENTO NO TRABALHO EM UTIS}

As UTIs ocupam áreas hospitalares destinadas ao atendimento de pacientes críticos que necessitem de cuidados complexos e especializados, onde concentram recursos humanos e materiais para o atendimento de pacientes graves que exigem assistência permanente, além da utilização de recursos tecnológicos apropriados para a observação e monitorização 
contínua das condições vitais e para a intervenção em situações de descompensações (GOMES, 1998; PADILHA; KIMURA, 2000).

Em virtude da constante expectativa de situações de emergência, da alta complexidade tecnológica e da concentração de pacientes graves, sujeitos a mudanças súbitas no estado geral, o ambiente de trabalho caracteriza-se como estressante e gerador de uma atmosfera emocionalmente comprometida, tanto para os profissionais como para os pacientes e seus familiares (KOIZUMI et al., 1979).

O trabalho dos profissionais de saúde é frequentemente considerado por quem o desempenha como desgastante e promotor de diversas patologias, além de contribuir para um desequilíbrio emocional e provocar queda no desempenho profissional (LANGE; FIORELLI, 2009). Na UTI, a equipe multiprofissional convive com outros fatores desencadeadores de estresse, tais como: a dificuldade de aceitação da morte; a escassez de leitos e equipamentos; escassez de recursos humanos e; tomada de decisões conflitantes relacionadas com a seleção dos pacientes que serão atendidos (PADILHA; KIMURA, 2000).

Alguns autores apontam outras causas de sofrimento de profissionais de saúde atuantes em UTIs: A sobrecarga de trabalho; a falta de tempo, a supremacia dada a rotina de trabalho em relação à interação com o familiar do paciente, a falta de treinamento, solicitações dos pacientes em estágio terminal, etc., constituem os aspectos negativos relacionados à função. Outro aspecto estudado que interfere negativamente no profissional são as situações com alta demanda emocional, como a piora das condições clínicas do paciente ou a identificação das fases do processo de morrer (SANTOS; SILVA, 2006; SUSAKI et al., 2006).

De acordo com Leite e Vila (2005) o estresse e a frustração do grupo de trabalho de UTIs se referem também à estrutura. Segundo os autores, através dos relatos dos profissionais foi possível apontar que a falta de recursos materiais, dificulta e interfere na qualidade da assistência prestada ao paciente crítico.

Esses são alguns dos dilemas éticos e profissionais vivenciados cotidianamente pela equipe que atua em terapia intensiva. Alguns participantes relataram que se sentem desmotivados, e isso produz relações de trabalho inadequadas. Essas situações criam tensão entre os profissionais e, em geral, influenciam, negativamente, a qualidade da assistência prestada aos clientes (LEITE; VILA, 2005).

Como citado previamente na UTI estudada o abastecimento de insumos não ocorre de forma sistemática e é dependente de um almoxarifado central, sem o controle direto dos profissionais da assistência. Conhecer a percepção dos colaboradores (médicos, enfermeiros, fisioterapeutas, auxiliares de enfermagem e técnicos de enfermagem) sobre a incidência de IRAS e sua relação com a falta de materiais foi o ponto de partida para este trabalho.

\section{METODOLOGIA}

\subsection{DESCRIÇÃO DA PESQUISA}

A pesquisa é de natureza exploratória, pois tem por objetivo conhecer a variável de estudo tal como se apresenta, seu significado e o contexto onde ela se insere (PIOVESAN; TEMPORINI, 1995) e de abordagem qualitativa, uma vez que não procurou enumerar e/ou medir os eventos estudados, nem emprega instrumental estatístico na análise dos dados, envolve a obtenção de dados descritivos sobre pessoas, lugares e processos interativos pelo contato direto do pesquisador com a situação estudada, procurando compreender os fenômenos segundo a perspectiva dos sujeitos, ou seja, dos participantes da situação em estudo (GODOY, 1995, p.58).

A estratégia empregada foi estudo de caso, que segundo Voss e colaboradores (2002) é uma história de um fenômeno passado ou atual, elaborada a partir de múltiplas fontes de provas, que pode incluir dados da observação direta e entrevistas sistemáticas, bem como pesquisas em arquivos públicos e privados.

Segundo Campos (2004), no universo das pesquisas qualitativas, a escolha de método e técnicas para a análise de dados deve proporcionar um olhar multifacetado sobre a totalidade dos dados recolhidos no período de coleta.

Portanto, o presente trabalho trata-se de um estudo de caso onde a análise documental das planilhas de insumos, notificações de IRAS e uso de medicamentos foram essenciais para corroborar com a análise de conteúdo das respostas dos entrevistados.

\subsection{PROTOCOLO DE PESQUISA}

O protocolo de pesquisa, para a estratégia de estudo de caso, foi baseado nos seguintes itens:

\subsubsection{Definição da Unidade de Análise}

O local foi a UTI de Clínica Médica de Emergência composta por 14 leitos de um hospital terciário 
público estadual, atuante como hospital de ensino de grande porte, localizado na cidade de São Paulo. Vale ressaltar que durante o período estudado a ocupação dos leitos era de $100 \%$, por se tratar de uma UTI de Emergências Clínicas do Pronto Socorro de um Hospital Público Terciário de Referência. Isso significa que a assistência no período foi contínua e não havia períodos de redução de abastecimento devido a leitos desocupados.

\subsubsection{Profissionais Entrevistados e Múltiplas Fontes de Evidência}

Para a escolha dos 23 entrevistados, os critérios foram profissionais permanentes e concursados para o cargo no setor (excluindo-se residentes médicos temporários e estagiários do curso de enfermagem), além de atuantes na assistência direta ao paciente, cujo tempo médio de exercício profissional na instituição e na UTI com no mínimo de seis anos.

Foram analisadas as planilhas de registro das notificações laboratoriais de bactérias multirresistentes, fornecidas pelo Serviço de CCIH. Os insumos utilizados pelos profissionais, para isolamento de contato, foram levantados através de planilha cedida pelo setor de insumos e os registros de dispensação mensal de antibióticos, administrados aos pacientes, no ano de 2012 e 2013, que foram cedidos pela farmácia hospitalar.

A observação participante foi realizada pelo fato de que uma das autoras é enfermeira chefe da UTI. Por meio das conversas informais, obtidas durante a condução da pesquisa, o pesquisador poderá obter evidências não perceptíveis na análise de documentos (FREITAS; JABBOUR, 2011). Este método de pesquisa vem mostrar a possibilidade da utilização do conhecimento dos participantes, ou seja, dos sujeitos envolvidos na pesquisa como agente colaborador e capaz de compor e implementar mudanças de suas atividades práticas, o que gera satisfação, possivelmente superior às pesquisas convencionais que apresentam relatórios e dados, sem o comprometimento dos resultados (GRITTEM et al., 2008).

\subsubsection{Período de Realização}

Trata-se de um estudo transversal e retrospectivo baseado em registros do serviço no passado, e é seguido adiante a partir daquele momento até o presente quando realizadas as entrevistas. É fundamental que haja credibilidade nos dados de registros, coletados de fontes primárias das supracitadas planilhas e entrevistas foram obtidos no período compreendido entre janeiro de 2012 a julho de 2013. Estudos transversais são estudos em que a exposição ao fator ou causa está presente ao efeito no mesmo momento ou intervalo de tempo analisado. Portanto, esse modelo apresenta-se como uma fotografia ou corte instantâneo que se faz numa população por meio de uma amostragem, examinando-se nos integrantes da casuística ou amostra, a presença ou ausência da exposição e a presença ou ausência do efeito. Possui como principais vantagens o fato de serem de baixo custo, e por praticamente não haver perdas de seguimento (HOCHMAN et al., 2005).

\subsubsection{Obtenção de Validade Interna}

Para a execução do estudo, este projeto seguiu as determinações do Conselho Nacional de Saúde, de acordo com a Resolução CNS no 466 (2012), sendo aprovado com o Parecer Consubstanciado $n^{\circ}$ 928557, registro online 12900 na Plataforma Brasil. Também obteve a apreciação e autorização da comissão de ética interna do hospital (CAAE 39216814.1.0000.0068), denominada Comissão de Ética para Análise de Projetos de Pesquisa (CAPPesq).

\subsubsection{Roteiro de Entrevista}

A entrevista tem sido um procedimento de coleta de dados amplamente utilizado em pesquisa em ciências humanas. Para alguns, ela é designada como um método; para outros, um instrumento de pesquisa; ou, ainda, uma técnica (TRIVIÑOS, 1987). As falhas na assistência por falta de material causam também uma instabilidade e insegurança no profissional da saúde e para que essas falhas sejam também compreendidas sob o ponto de vista destes profissionais foi aplicado um questionário baseado em um roteiro semiestruturado aos funcionários da UTI, conforme o construto elaborado com base em diversas fontes da literatura (Quadro 2). Independente da abordagem teórica adotada, principalmente quando a entrevista é do tipo semiestruturada, são necessários cuidados que envolvem questões da linguagem e o roteiro a ser utilizado necessita ser planejado cuidadosamente (MANZINI, 2003).

Pode-se dizer, então, que, para se obter determinada resposta, é preciso fazer a pergunta correspondente. 
Além disso, para se obter "boas" respostas é preciso fazer-se "boas" perguntas. Mas para fazer "boas perguntas", ou perguntas pertinentes e de interesse, é preciso conhecer com antecipação as possíveis respostas, que consistiriam no "Universo de Respostas". Quanto melhor o conhecimento do Universo de Respostas, mais capacitado está o pesquisador em elaborar um bom instrumento de pesquisa. E ele será um bom instrumento, na medida em que for capaz de fazer boas perguntas (PIOVESAN; TEMPORINI, 1995).

Quadro 2: Referências associadas aos pressupostos do instrumento de pesquisa.

\begin{tabular}{|c|c|c|c|}
\hline PRESSUPOSTOS & $\begin{array}{l}\text { AUTOR(ES) E } \\
\text { ANO DE PUBLICAÇÃO }\end{array}$ & PASSAGEM DO TEXTO & $\begin{array}{l}\text { PERGUNTA } \\
\text { ELABORADA }\end{array}$ \\
\hline $\begin{array}{l}\text { Uso de Equipamentos } \\
\text { de Proteção Individual } \\
\text { (EPIs) no cuidado. }\end{array}$ & BATISTA (2004) & $\begin{array}{l}\text { O EPI (Equipamento de } \\
\text { Proteção Individual) é uma } \\
\text { barreira mecânica. }\end{array}$ & $\begin{array}{l}\text { 1. Quanto ao uso de EPIs, quais } \\
\text { os usualmente disponíveis na } \\
\text { sua unidade? }\end{array}$ \\
\hline $\begin{array}{l}\text { Falta de materiais } \\
\text { para a execução do } \\
\text { trabalho em uma UTI. }\end{array}$ & $\begin{array}{l}\text { FIGUEIREDO; ZUFFI } \\
\text { (2012) }\end{array}$ & $\begin{array}{l}\text { Dificuldades em relação à falta } \\
\text { de material e local adequado } \\
\text { para realização do curativo foi } \\
\text { apontado em mais de } 50 \% \text { das } \\
\text { entrevistas aplicadas pelos } \\
\text { autores. }\end{array}$ & $\begin{array}{l}\text { 2. Quais os materiais cujas } \\
\text { faltas são observadas com mais } \\
\text { frequência em sua unidade? }\end{array}$ \\
\hline $\begin{array}{l}\text { Relacionar risco de } \\
\text { IRAS com falta de } \\
\text { insumos e EPIs. }\end{array}$ & FERREIRA et al. (2000). & $\begin{array}{l}\text { Enfermeiros e médicos ainda } \\
\text { realizam procedimentos } \\
\text { sem o uso de EPIs, pela não } \\
\text { disponibilização no suprimento } \\
\text { de materiais de segurança pela } \\
\text { direção do hospital. }\end{array}$ & $\begin{array}{l}\text { 3. Quais as dificuldades na } \\
\text { prestação de assistência } \\
\text { relacionadas com a falta de } \\
\text { material em sua unidade? }\end{array}$ \\
\hline $\begin{array}{l}\text { Percepção sobre as } \\
\text { razões das falhas de } \\
\text { abastecimento. }\end{array}$ & LANNA (2010) & $\begin{array}{l}\text { A falta de materiais tem muitos } \\
\text { motivos (recursos financeiros } \\
\text { até falta de atenção gerencial). }\end{array}$ & $\begin{array}{l}\text { 4. Por quais motivos você acha } \\
\text { que os materiais faltam? }\end{array}$ \\
\hline $\begin{array}{l}\text { Participação } \\
\text { no processo de } \\
\text { aquisição e gestão de } \\
\text { suprimentos do setor. }\end{array}$ & OLIVEIRA; CHAVES (2009) & $\begin{array}{l}\text { A classificação de materiais } \\
\text { em diferentes grupos ou } \\
\text { classes possibilita estabelecer } \\
\text { instrumentos de planejamento e } \\
\text { controle adequado. }\end{array}$ & $\begin{array}{l}\text { 5. Sugestões que você daria } \\
\text { ao setor responsável pelas } \\
\text { compras, para a melhoria do } \\
\text { desempenho em sua unidade? }\end{array}$ \\
\hline $\begin{array}{l}\text { Participação dos } \\
\text { colaboradores } \\
\text { no processo de } \\
\text { abastecimento de } \\
\text { insumos na UTI. }\end{array}$ & $\begin{array}{l}\text { HONÓRIO; } \\
\text { ALBUQUERQUE (2005) }\end{array}$ & $\begin{array}{l}\text { Falta de envolvimento da equipe } \\
\text { de enfermagem com a Gestão } \\
\text { de Recursos Materiais (GRM). }\end{array}$ & $\begin{array}{l}\text { 6. Na falta de um item, qual a } \\
\text { atitude da equipe de trabalho? }\end{array}$ \\
\hline $\begin{array}{l}\text { Percepção da } \\
\text { biossegurança e } \\
\text { importância de } \\
\text { EPIs no cuidado } \\
\text { e assistência ao } \\
\text { paciente. }\end{array}$ & OLIVEIRA; CHAVES (2009) & $\begin{array}{l}\text { A preocupação com as medidas } \\
\text { de biossegurança foi observada } \\
\text { no hospital, principalmente, } \\
\text { quanto às orientações para a } \\
\text { utilização dos EPIs. }\end{array}$ & $\begin{array}{l}\text { 7. Quais os itens de consumo } \\
\text { mais importantes na sua } \\
\text { atividade diária? }\end{array}$ \\
\hline $\begin{array}{l}\text { Percepção da } \\
\text { qualidade de insumos } \\
\text { e EPIs na assistência. }\end{array}$ & $\begin{array}{l}\text { HONÓRIO; } \\
\text { ALBUQUERQUE (2005) }\end{array}$ & $\begin{array}{l}\text { A equipe alimenta a expectativa } \\
\text { de que os materiais adquiridos } \\
\text { atendam às reais necessidades } \\
\text { da assistência. }\end{array}$ & $\begin{array}{l}\text { 8. Qual sentimento você tem } \\
\text { quando deixa de prestar } \\
\text { assistência pela falta de } \\
\text { qualidade dos insumos? }\end{array}$ \\
\hline $\begin{array}{l}\text { Percepção da forma } \\
\text { de aquisição dos } \\
\text { insumos e EPls de } \\
\text { serviço. }\end{array}$ & OLIVEIRA; CHAVES (2009) & $\begin{array}{l}\text { Importância da avaliação } \\
\text { feita pelas enfermeiras } \\
\text { e estabelecimento de } \\
\text { especificações técnicas exatas } \\
\text { em relação àquilo que se deseja } \\
\text { adquirir. }\end{array}$ & $\begin{array}{l}\text { 9. O que poderia melhorar em } \\
\text { relação à aquisição de insumos } \\
\text { em sua unidade? }\end{array}$ \\
\hline
\end{tabular}


Uma apreciação final sobre o tema permitiu que cada entrevistado falasse livremente, registrado como observações no final do roteiro aplicado, representada pela questão de número $10 \mathrm{do}$ roteiro, a fim de conhecer o real impacto da falta de um insumo ou EPI na condução do trabalho de assistência, para a garantia da saúde do paciente. Nesta questão o entrevistado deveria apontar uma situação crítica (que mais o incomodava) no desempenho de suas funções relacionadas à utilização de materiais.

Para cada uma das respostas foi realizada a análise de conteúdo que, segundo Bardin (2009) pode ser descrita como um conjunto de técnicas de investigação que, através de uma descrição objetiva, sistemática e quantitativa do conteúdo manifesto das comunicações, tem por finalidade a interpretação destas mesmas comunicações.

\section{RESULTADOS E CONCLUSÕES}

\subsection{FALTA DE INSUMOS E OCORRÊNCIA DE IRAS ENTRE 2012 E 2013}

O número total de trabalhadores da UTI entrevistados foi de 23 e o número por categoria profissional é apresentado na Tabela 1 .

QUADRO 02 - ESTILO DE LIDERANÇA PREDOMINANTE DO LÍDER DE ACORDO COM O SUPERIOR DOS LÍDERES

\begin{tabular}{|l|c|}
\hline PROFISSÃO & NÚMERO DE PROFISSIONAIS \\
\hline Auxiliar de enfermagem & 7 \\
Técnico de enfermagem & 4 \\
Fisioterapeuta & 2 \\
Enfermeiro & 8 \\
Médico & 2 \\
Total & $\mathbf{2 3}$ \\
\hline
\end{tabular}

Dos participantes, $100 \%$ relataram que as luvas, as máscaras e os aventais são disponíveis na unidade em boa parte do tempo. Porém, o item mais apontado como "em falta" foi o avental de isolamento, o qual não é solicitado pela enfermagem ao almoxarifado, mas sim entregues pela rouparia, que apresentou alguns intervalos para entrega dos enxovais em perí- odos longos, devido à terceirização da empresa que realizava a higiene destes itens.

A análise documental das planilhas de solicitações de materiais mostra que no segundo semestre de 2012, entre os meses de agosto e novembro de 2012, houve um período crítico de desabastecimento de aventais impermeáveis de mangas longas (não estéril) para a UTI (Figura 1).

FIGURA 1. DISTRIBUIÇÃO DE FORNECIMENTO DE AVENTAIS NO PERÍODO DE JULHO DE 2012 A JUNHO DE 2013.

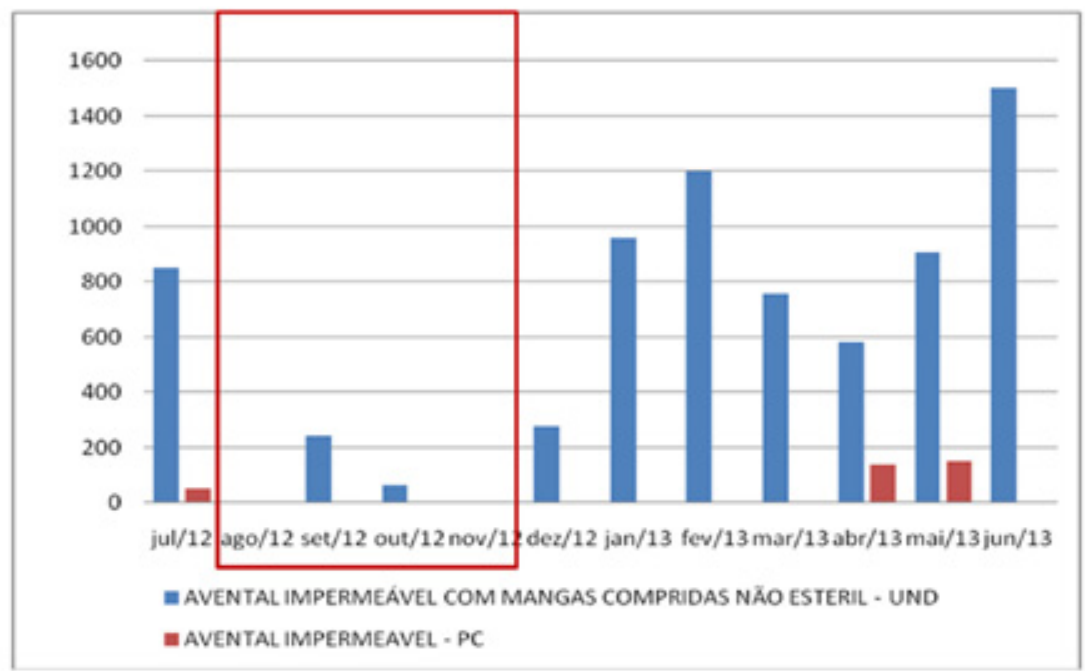

Fonte: Elaborada pelos autores com base na planilha de insumos. 
Ainda no mesmo período, nota-se que, entre agosto e dezembro de 2012, a falta de abastecimento de aventais parece ter sido compensada pelo aumento de abastecimento de luvas (Figura 2).

FIGURA 2. FORNECIMENTO DE LUVAS NO PERÍODO DE AGOSTO A DEZEMBRO DE 2012.

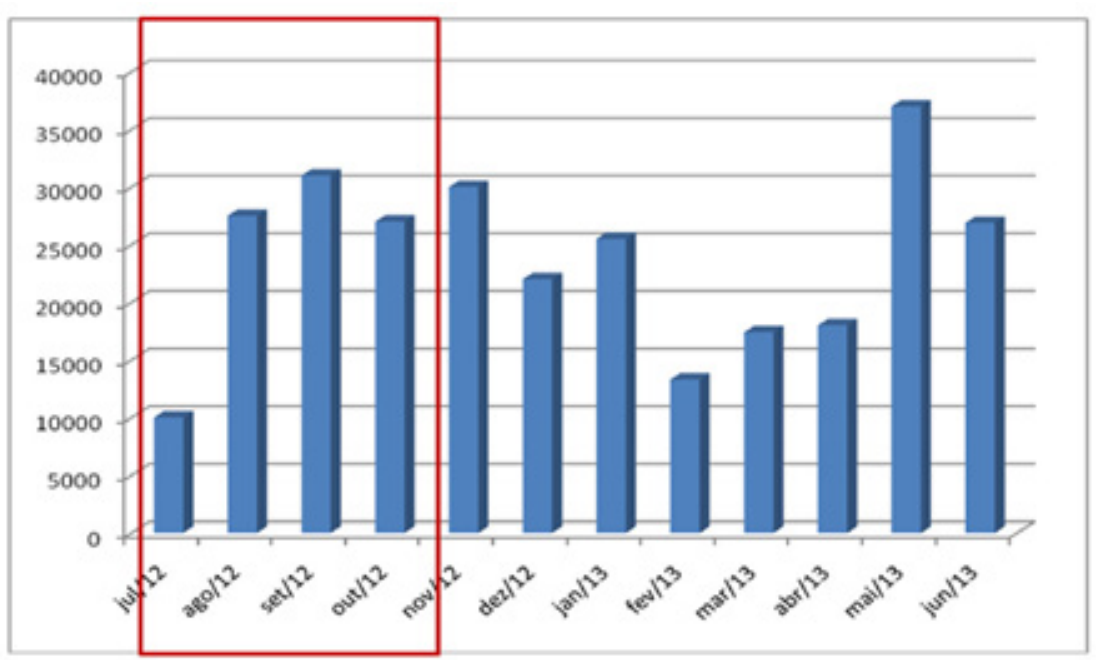

Fonte: Elaborada pelos autores com base na planilha de insumos.

Após o mês de agosto (período de falta de aventais), houve um aumento do número de prescrições de dois antibióticos, a Colistina e o Carbapenem (Figura 3), muito empregados no controle de in- fecção hospitalar causado pela bactéria Klebisiella Pneumoniae Carbapenemase resistente (KPC), comum em casos de infeccções hospitalares (ANVISA, 2014).

\section{FIGURA 3. DISTRIBUIÇÃO DE ANTIBIÓTICOS DEVIDO À INFECÇÃO POR KPC (KLEBISIELLA PNEUMONIAE CARBAPENEMASE RESISTENTE) NO PERÍODO ESTUDADO, 2012.}

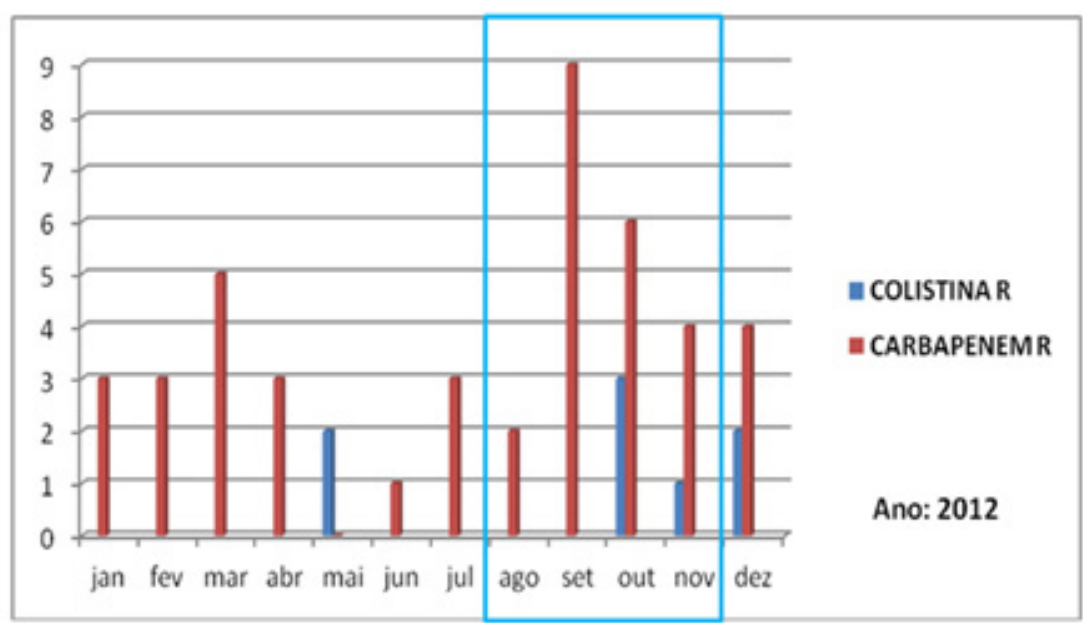

Fonte: Elaborada pelos autores com dados cedidos pela farmácia hospitalar e SCCIH.

Segundo os entrevistados no primeiro semestre de 2012 foi detectada a ocorrência da falta de avental, devido ao problema de abastecimento do fornecedor para o almoxarifado central. Foi observado que a falta de abastecimento de aventais foi compensada pelo aumento de consumo de luvas descartáveis, fato esse que não evitou o aumento de casos de infecção hospitalar, ao contrário, observa-se que após o mês de agosto de 
2012 houve um elevado número de prescrições de antibióticos indicados para o tratamento por infecção e/ ou colonização pela bactéria KPC nos pacientes, de acordo com a Figura 5, onde se observa (no mês de setembro) a elevação de prescrição dos antibióticos Colistina e Carbapenem.

No mesmo período, devido às infecções por microrganismo multirresistente, a Klebsiella pneumoniae (KPC), ocorreu o pior dos desfechos, como óbitos de pacientes, onde foram notificados nos relatórios da SCCIH um total de 44 pacientes com infecção por $\mathrm{KPC}$, sendo $18 \mathrm{com}$ desfecho em óbitos. O próprio surto interno da KPC e consequente mortes gerou estresse na equipe de atendimento, conforme apontado pelos entrevistados.

No mesmo período de aumento de infecções, luvas foram utilizadas de maneira inapropriada, como garrote para punção venosa e em outras situações (devido à falta do tamanho ideal) faziam com que os profissionais aumentassem o consumo, pois as mesmas rasgavam ao serem colocadas em mãos maiores do que a numeração real. E ainda havia o risco para acidentes, pois luvas muito grandes em mãos muito pequenas reduzem a área de contato e dificultam a precisão da atividade a ser realizada, podendo sim estar relacionada com a incidência de infecções.

\subsection{PERCEPÇÃO DOS PROFISSIONAIS DA UTI}

Em certa medida, não há como desconsiderar que a organização do trabalho e a gestão em saúde foram cronologicamente atravessadas por traços do modelo taylorista, com a fragmentação do conhecimento e das práticas (organização parcelar do trabalho, especializações dentro de cada categoria profissional, centralização no modelo biomédico), burocratização, normatizações técnicas, sistemas de controle de produção de serviços e procedimentos, mecanismos de alienação e imobilidade, que até hoje seguem exercendo profunda influência nos conceitos e processos de trabalho (RIBEIRO et al., 2004). Muitos estudos reforçam a concepção do significado do trabalho, alertando a respeito das causas do sofrimento psíquico, pois devem ser buscadas nas atividades humanas geradoras de significado, e uma delas é o trabalho (CODO, 2002). A seguir, as respostas são apresentadas de acordo com o roteiro semiestruturado. A primeira questão se refere à percepção sobre o abastecimento de EPIs, cujas respostas estão enumeradas na Tabela 2.

TABELA 2. RESPOSTAS SOBRE A PERCEPÇÃO DOS PROFISSIONAIS SOBRE EPIS SEMPRE DISPONIVEIS.

\begin{tabular}{|l|c|c|}
\hline EPIS & (N) & (\%) \\
\hline Luvas de procedimento e estéreis & 19 & 83 \\
Avental & 19 & 83 \\
Máscara & 17 & 74 \\
Óculos & 14 & 61 \\
Gorro & 5 & 22 \\
Máscara $\mathrm{n}^{\circ} 95$ & 1 & 4 \\
Álcool gel & 1 & 4 \\
\hline
\end{tabular}

Os materiais cujas faltas são observadas com mais frequência na UTI está apresentada na Figura 4 
FIGURA 4. DISTRIBUIÇÃO DOS ITENS EM FALTA QUANTO À PERCEPÇÃO DO COLABORADOR.

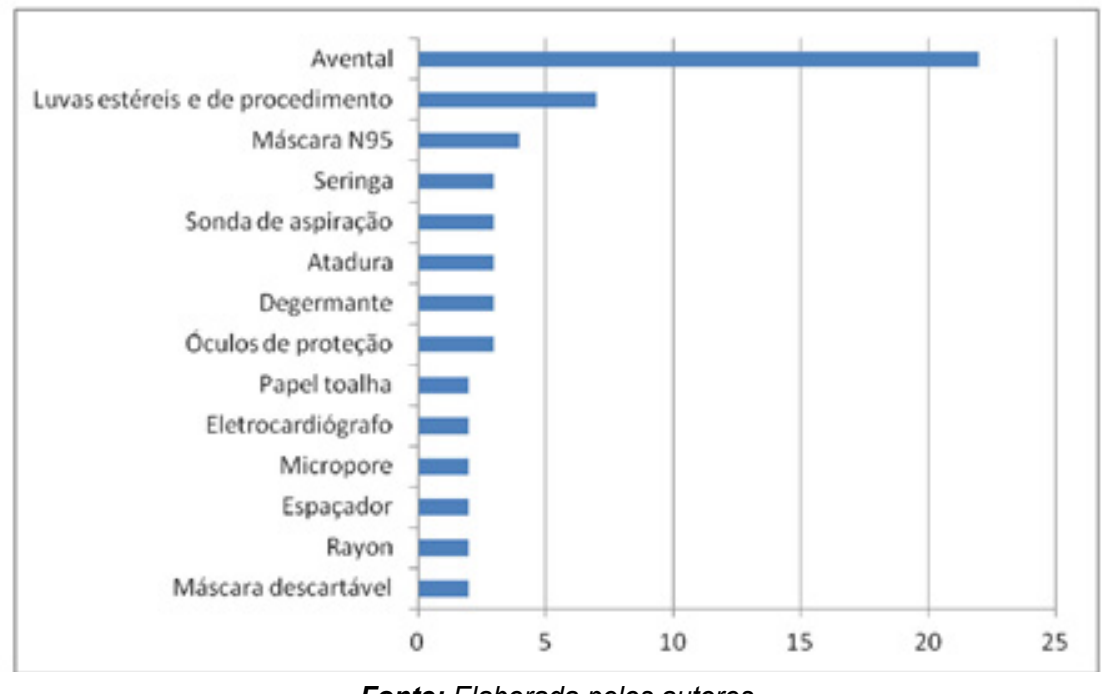

Fonte: Elaborada pelos autores

Em relação às questões 1 e 2 , embora os aventais e luvas ( $83 \%$ ) serem os itens mais lembrados como sempre disponíveis pelos profissionais (Tabela 2) os mesmos são considerados mais lembrados pela falta. A falta de avental foi lembrada em $95 \%$ das respostas, seguido da falta de luvas (34\%) conforme demonstra a Figura 6. A particularidade do item avental é que a instituição substituiu o avental descartável pelo avental de tecido em 2012. Este passou a ser higienizado por empresa terceirizada e distri- buído pelo setor de rouparia, por meio de pedido telefônico, o que inviabilizou a entrega em muitas ocasiões, segundo os colaboradores. Quanto às dificuldades na prestação de assistência, relacionadas com a falta de material na UTI, a Tabela 3 apresenta a frequência de tais percepções. A percepção sobre a demora no atendimento e o temor da infecção cruzada são as dificuldades mais percebidas nas respostas dadas pelos profissionais, conforme a Tabela 3 (26\%, respectivamente).

TABELA 3. PERCEPÇÃO DOS PROFISSIONAIS QUANTO À DIFICULDADE NA ASSISTÊNCIA POR FALTA DE MATERIAIS.

\begin{tabular}{|l|c|c|}
\hline PERCEPÇÃO OBSERVADA & (N) & (\%) \\
\hline Demora no atendimento & 6 & 26,0 \\
Infecção cruzada & 6 & 26,0 \\
Falta de abastecimento de materiais & 4 & 17,4 \\
Precaução de contato & 2 & 8,7 \\
Reposição de material & 2 & 8,7 \\
Qualidade da assistência & 1 & 4,3 \\
Dificuldade em organizar o atendimento & 1 & 4,3 \\
Exame do paciente & 1 & 4,3 \\
Medicação & 1 & 4,3 \\
Adaptação de equipamentos & 1 & 4,3 \\
Não há falta & 1 & 4,3 \\
Uso inadequado & 1 & 4,3 \\
\hline
\end{tabular}

A demora no atendimento pode ser crucial para a sobrevivência do paciente ao ser admitido em uma UTI e a falta de abastecimento de materiais (apon- tada como $17 \%$ das respostas) pode estar associada na busca do material faltante no setor de almoxarifado ou em outras unidades intra-hospitalares. A 
percepção sobre quais os motivos da falta de materiais indicam que o desperdício e improvisação são percepções mais lembradas, seguidas de falhas administrativas (Tabela 4).

TABELA 4. PERCEPÇÃO DOS PROFISSIONAIS SOBRE A RAZÃO DA FALTA DE MATERIAIS.

\begin{tabular}{|l|c|c|}
\hline PERCEPÇÃO OBSERVADA & (N) & (\%) \\
\hline Desperdício de material e improvisação & 8 & 34,8 \\
Má administração e planejamento & 7 & 30,4 \\
Previsão inadequada do consumo & 7 & 30,4 \\
Falta de pessoal treinado na entrega & 7 & 30,4 \\
Falta de organização e controle do estoque & 7 & 30,4 \\
Falta de prioridades & 1 & 4,3 \\
Verbas insuficientes & 1 & 4,3 \\
Não sabe & 1 & 4,3 \\
Não há falta & 1 & 4,3 \\
\hline
\end{tabular}

A improvisação e desperdício de material foram observados como mais registrados na Tabela $4(34,8 \%)$. Esses dados corroboram com outros estudos que apontam que independentemente dos motivos, a realidade é que a carência de recursos financeiros determina a diminuição ou mesmo a supressão da aquisição de materiais, fazendo com que os profissionais de enfermagem utilizem o artifício da improvisação na assistência para substituir o ma- terial ausente (VAGHETTI et al., 2011). Contudo, a contínua busca pela eficiência tecnocientífica requer uso racional de recursos, sem aquisição de arranjos ou improvisações indevidas de materiais e equipamentos que possam provocar práticas inconsequentes em serviços de saúde (OLIVEIRA et al., 2012). Em relação às sugestões dadas, para a melhoria do desempenho na UTI, o controle de estoque foi o mais apontado pelos profissionais (Tabela 5).

TABELA 5. SUGESTÕES DOS PROFISSIONAIS PARA MELHORIAS NO ABASTECIMENTO DE INSUMOS.
\begin{tabular}{|l|c|c|}
\hline SUGESTÕES APONTADAS & (N) & (\%) \\
\hline Controle da utilização e estoque & 14 & 60,8 \\
Planejamento e organização & 9 & 39,1 \\
Educação p/ uso consciente & 7 & 30,4 \\
Controle da distribuição e agilidade & 5 & 21,7 \\
Horário fixo de entrega e reposição pelo almoxarifado & 4 & 17,4 \\
Previsão do consumo & 4 & 17,4 \\
Almoxarifado 24 horas & 2 & 8,7 \\
Adequação do setor de compras & 2 & 8,7 \\
Compromisso & 1 & 4,3 \\
Comunicação efetiva & 1 & 4,3 \\
Visualização do estoque & 1 & 4,3 \\
Objetivos claros & 1 & 4,3 \\
Confecção de kits para procedimentos & 1 & 4,3 \\
\hline
\end{tabular}

Os respondentes discorreram sobre a necessidade de um maior planejamento e organização do abastecimento e sobre o controle do uso e estoque pelo setor. Quanto a essa visão, Paschoal e Castilho (2010) afirmam que o
SGM, além de aprimorar o controle de material, devido à agilidade e praticidade, induz o profissional refletir suas atitudes no dia a dia do trabalho, devido a sua responsabilização no processo. Outro aspecto apontado 
pelos autores é que a partir do momento que o usuário adquire confiança no sistema de gestão de material, ele deixa de solicitar o material em abundância para se sentir seguro ao prestar assistência ao paciente.

Como apontado na questão 3 , sobre a percepção da demora no atendimento ao paciente, por falta de algum material, esse atraso está relacionado no deslocamento de algum membro da equipe na busca do item em outra unidade hospitalar, tal observação está evidente na resposta 6 , onde $52 \%$ dos profissionais afirmam se deslocar para emprestar materiais de outra unidade (Tabela 6).

TABELA 6. MEDIDAS APONTADAS NA FALTA DE SUPRIMENTOS COMO TOMADA DE DECISÃO.

\begin{tabular}{|l|c|c|}
\hline TOMADA DE DECISÃO & (N) & (\%) \\
\hline Empresta de outra unidade & 12 & 52,2 \\
Comunica a chefia, a SCCIH e a escriturária & 9 & 39,1 \\
Substitui por similar ou improvisa & 7 & 30,4 \\
Solicita ao almoxarifado/rouparia & 7 & 30,4 \\
Não realiza o procedimento & 2 & 8,7 \\
\hline
\end{tabular}

Para realização da assistência segura ao paciente é necessário o cumprimento de regras e técnicas específicas para cada procedimento; cada ação requer a utilização de vários materiais, portanto em cada unidade de assistência deve existir o mínimo de materiais de consumo diário, necessários para realização correta dos procedimentos, sem que o profissional de saúde saia de sua unidade em busca de insumos, durante a assistência ao paciente.

Segundo Mendes e Castilho (2009), na tentativa de minimizar esse problema ocorrem empréstimos de materiais entre algumas unidades hospitalares, mas nem sempre isso é possível, porque na maioria das vezes há falta de material em todas as unidades, devido ao déficit no estoque do almoxarifado.

Luvas e avental ainda são percebidos como os itens primordiais na assistência, devido ao aumento dos casos de IRAS entre agosto e dezembro de 2012, quando a falta de aventais foi notada pela equipe da UTI (Figura 6). Ainda a percepção é de que esses dois itens são primordiais para evitar casos de IRAS (Tabela 7).

TABELA 7. ITENS CONSIDERADOS ESSENCIAIS OU MAIS IMPORTANTES PARA O PROFISSIONAL.

\begin{tabular}{|l|c|c|}
\hline TIPO DE ITEM & (N) & (\%) \\
\hline Luvas de procedimento e estéreis & 16 & 69,5 \\
Avental de isolamento & 16 & 69,5 \\
Máscara & 10 & 43,5 \\
Todos & 6 & 26,0 \\
Óculos & 4 & 17,4 \\
Swab alcoólico/álcool & 3 & 13,0 \\
Soro fisiológico & 3 & 13,0 \\
Seringas & 3 & 13,0 \\
Agulhas & 2 & 8,7 \\
Filtro HME & 1 & 4,3 \\
Degermante/Clorexidina & 1 & 4,3 \\
Gaze & 1 & 4,3 \\
Ataduras & 1 & 4,3 \\
Lençóis & 1 & 4,3 \\
\hline
\end{tabular}


Com relação ao sentimento ao prestar assistência, pela falta de qualidade dos insumos, impotência $(21,7 \%)$, frustração $(17,4 \%)$ e revolta (13\%), são as sensações mais relatadas pelos profissionais perante a falta de insumos (Tabela 8). Questões nevrálgicas como essa são comuns na realidade dos hospitais públicos e devem ser cuidadosamente consideradas, sob a pena de serem mais um fator de estresse para a equipe de enfermagem, que já trabalha em situações que envolvem os limites da vida, e de descrença desses hospitais (VAGHETTI et al., 2011).

TABELA 8. SENTIMENTO RELATADO PELOS COLABORADORES PELA PERCEPÇÃO DA FALTA DE MATERIAL.

\begin{tabular}{|l|c|c|}
\hline SENTIMENTO MANIFESTO & (N) & (\%) \\
\hline Impotência & 5 & 21,7 \\
Frustração & 4 & 17,4 \\
Raiva/revolta & 3 & 13,0 \\
Não se envolve emocionalmente & 3 & 13,0 \\
Não realiza o procedimento & 3 & 13,0 \\
Assistência prejudicada & 3 & 13,0 \\
Tristeza & 2 & 8,7 \\
Inconformismo & 1 & 4,3 \\
Indignação & 1 & 4,3 \\
Desânimo & 1 & 4,3 \\
Preocupação & 1 & 4,3 \\
\hline
\end{tabular}

Percebe-se um grupo de profissionais que não demonstram qualquer relação emocional frente ao problema (13\%) e outros que não cumprem o procedimento (13\%) sem buscar uma solução. Outros relacionam a falta de materiais com a assistência prejudicada $(13 \%)$.

O profissional de saúde, em seu cotidiano lida com problemas durante a convivência diária junto a pacientes, familiares e colegas e que tem contribuído para gerar situações de estresse de difícil resolução. O sentimento gerado por estas situações, muitas vezes, se traduz em impotência, frustração e revolta (KOVÁCS, 2010). Na percepção dos profissionais para sugestões de melhorias, na aquisição de insumos da UTI, as respostas apontaram o planejamento institucional como principal foco $(56,5 \%)$ (Tabela 9).

TABELA 9. SUGESTÕES DE MELHORIAS A PONTADAS PELOS RESPONDENTES.

\begin{tabular}{|l|c|c|}
\hline SUGESTÕES DE MELHORIAS & (N) & (\%) \\
\hline Planejamento institucional & 13 & 56,5 \\
Administração direta das compras e estoque & 9 & 39,1 \\
Organização do atendimento & 9 & 39,1 \\
Redução do desperdício & 6 & 26,0 \\
Compromisso e conscientização dos profissionais & 3 & 13,0 \\
Controle diário dos estoques & 3 & 13,0 \\
Agilidade na reposição do material & 1 & 4,3 \\
Nada & 1 & 4,3 \\
\hline
\end{tabular}

Observa-se de maneia geral que a falta de material foi o item mais lembrado $(65,2 \%)$ e que os profissio- nais apontaram em mais de uma resposta que a falta de avental e o medo do risco de infecção cruzada 
(Tabela 10) são fatores que esses profissionais associam à falha na assistência e consequente aumento de casos de IRAS, decorrente do período de agosto a dezembro de 2012.

TABELA 10. RESPOSTAS QUANTO AOS FATORES CONSIDERADOS CRÍTICOS DA FALTA DE ABASTECIMENTO.

\begin{tabular}{|l|c|c|}
\hline PONTOS CRÍTICOS & (N) & (\%) \\
\hline Falta de material & 15 & 65,2 \\
Desperdício/uso inadequado & 14 & 60,8 \\
Má qualidade do material & 4 & 17,4 \\
Perda de tempo & 2 & 8,7 \\
Assistência inadequada & 2 & 8,7 \\
Qualidade & 2 & 8,7 \\
Quantidade insuficiente & 2 & 8,7 \\
Indisponibilidade & 2 & 8,7 \\
Comunicação/informação & 1 & 4,3 \\
Nenhum & 2 & 8,7 \\
\hline
\end{tabular}

Outro fator que mais incomoda os profissionais de saúde foi o desperdício ou uso inadequado dos insumos $(60,8 \%)$. Os trabalhadores entendem que o desperdício tem se tornado uma prática inevitável, mesmo que não intencional, pois necessitam, cotidianamente, subutilizar e até inutilizar materiais para garantir o exercício do cuidado (VAGHETTI et al., 2011).

A qualidade do material disponível é indispensável para qualificar a assistência de enfermagem prestada, viabilizar cuidado ao paciente e a atuação da equipe dentro dos padrões desejáveis de segurança (OLIVEIRA; CHAVES, 2009). Dentre as reformulações necessárias em busca da qualidade do material oferecido está a melhora nas especificações dos materiais padronizados e não padronizados, sendo possível obter materiais de qualidade superior e consequentemente melhora da assistência oferecida (GARCIA et al., 2012).

A reposição contínua leva ao aumento da frequência das entregas dos materiais sincronizando-as com a demanda, reduzindo drasticamente os estoques (Barbieri \& Machline, 2006). A qualidade é um aspecto importante não apenas no teste de materiais durante o processo de compra, mas também durante o acompanhamento do uso destes materiais com visitas à segurança do paciente e da equipe (OLIVEIRA et al., 2012).

Através da adoção de medidas voltadas para educação permanente, a instituição deve estimular os profissionais com aulas, cursos, seminários e palestras com o objetivo de evidenciar a importância da adoção e implementação de medidas visando à proteção e segurança não só dos profissionais como também dos pacientes que estão sob seus cuidados. Além disso, é preciso que haja reformulação de políticas de prevenção de riscos ocupacionais, avaliação contínua da saúde dos trabalhadores e dos ambientes hospitalares (AMARAL et al., 2012).

No estudo apresentado por Roberto e Lira (2010), e também na opinião dos entrevistados, o ideal é que o processo de controle de materiais num hospital esteja sobre a responsabilidade técnica de um profissional preparado e que se conte com um número suficiente de pessoas, com formação adequada, de acordo com a complexidade e as funções desenvolvidas em cada processo.

Um ponto importante para ressaltar é que a Gestão de Suprimento de Materiais não tem recebido o devido trato profissional, desvalorizando-se o setor, por conseguinte a empresa hospitalar. Por vezes, delega-se essa responsabilidade a colaboradores não qualificados para o exercício da função. Os conceitos básicos, a linguagem técnica e os conhecimentos necessários devem ser adquiridos através de cursos especializados, o que resulta em ganhos de qualidade, produtividade, investimento no capital humano, eficiência e eficácia (ROBERTO; LIRA, 2010).

O levantamento dos insumos fornecidos no período de 2012 a 2013 demonstrou a falta de materiais 
essenciais ao longo de todo o ano, principalmente de insumos fundamentais para assistência ao cliente. A ocorrência de IRAS no período da falta de abastecimento de aventais em 2012 foi uma percepção marcante entre os profissionais e uma das lições aprendidas, que serviram como sinalizadores da falta de um insumo com os riscos de IRAS em uma UTI.

Ficou evidente ainda que o aumento do aporte de luvas, não tornou a falha de abastecimento do item "avental" aceitável como substituição por outro tipo de insumo, e que o desperdício de material (luvas) também causou desconforto e estresse nos profissionais.

O departamento de suprimento em serviços de saúde deve atender ao máximo a demanda de solicitações do profissional na assistência de uma UTI e, para isso, conhecer a percepção dos colaboradores a respeito do sistema, auxilia em melhorias e justificam o investimento em estoques específicos, viabilizando as soluções encontradas pela equipe para atingir os objetivos, a que se destinam as instituições de saúde.

Espera-se que os serviços de saúde tenham o mínimo de insumos para que o paciente receba a melhor assistência e os profissionais possam executar seus trabalhos guiados pelas boas práticas com os materiais básicos e de qualidade, para que desta forma, possam dedicar maior tempo à assistência do que nas soluções de problemas emergenciais do processo logístico de insumos, em improvisações, buscas ou reposições de materiais considerados essenciais.

\section{LIMITAÇÕES}

Uma limitação do estudo foi realizar uma pesquisa sobre a percepção dos próprios gestores de compras e funcionários do almoxarifado que, por outro lado, sofrem com as pressões ou reclamações dos profissionais de saúde da UTI, quando suas requisições não são atendidas.

\section{RECOMENDAÇÕES DE ESTUDO}

Como sugestão ou contribuição para a prática profissional, o planejamento da aquisição de materiais deve levar em conta a percepção dos colaboradores atuantes de uma UTI, como Steakholders envolvidos na operação logística e administrativa. A avaliação dos materiais e equipamentos, antes de sua aquisição, para que os profissionais que vão utilizá-los em sua prática profissional possam emitir a sua opinião sobre o produto, ajudaria na tomada de decisão da seleção de fornecedores no processo licitatório. Com esse enfoque, um novo estudo poderia ser desenvolvido como um aspecto prospectivo (iniciado no presente, e seguindo para o futuro), a fim de obter resultados de uma ação de intervenção, como na pesquisa-ação.

Desta forma, os profissionais de saúde se sentiriam mais participativos, confiantes e certos de que a execução de seu trabalho em uma UTI não trará danos adicionais aos pacientes e nem a si mesmo por falta de materiais.

\section{REFERÊNCIAS}

AGÊNCIA NACIONAL DE VIGILÂNCIA SANITÁRIA. Documento de referência para o Programa Nacional de Segurança do Paciente, 2014. Disponível em: http://bvsms.saude.gov.br/bvs/publicacoes/ documento_referencia_programa_nacional_seguranca.pdf. Acessado em: 5 nov. 2014.

. Medidas de prevenção de infecção relacionada à saúde, 2013. Disponível em: http://portal. ANVISA.gov.br/wps/content/ANVISA+Portal/ANVISA/Inicio/Servicos+de+Saude/Assunto+de+Interesse/ Aulas + Cursos + Cartazes + Publicacoes + e + Seminarios/Controle + de + Infeccao + em + Servicos + de + Saude/ Manuais. Acesso em: 8 nov. 2014.

. Resolução da Diretoria Colegiada n ${ }^{0} 42$ de 25 de outubro de 2010. Dispõe sobre a obrigatoriedade de disponibilização de preparação alcoólica para friç̧ão antisséptica das mãos, pelos serviços de saúde do País. Disponível em: http://www.anvisa.gov.br/hotsite/segurancadopaciente/documentos/rdcs/RDC\%20 N\%C2\%BA\%2042-2010.pdf. Acesso em: 12 dez. 2014. 
. Nota Técnica ANVISA no 01 de 17 de abril de 2013. Medidas de prevenção e controle de infecções por enterobactérias multirresistentes. Disponível em: http://portal.anvisa.gov.br/wps/wcm/connect/ ea4d4c004f4ec3b98925d9d785749fbd/Microsoft+Word+NOTA+T\%C3\%89CNICA+ENTEROBACTERI AS+17+04+2013(1).pdf?MOD=AJPERES. Acesso em: 15 dez. 2014.

AMARAL, M. H. S. P.; PINHEIRO, M. D. P.; LA CAVA, A. M. Riscos inerentes ao trabalho da equipe multiprofissional na Unidade de Terapia Intensiva. Acreditação, v.1, n.1: p. 29-45, 2012.

BARBIERI, J. C.; MACHLINE, C. (2006). Logística hospitalar: teoria e prática. São Paulo: Saraiva, 2006, p. 51-191.

BARDIN, L. Análise de conteúdo. Lisboa: Edições 70, 2009.

BATISTA, R. E. A. Módulo 1. Legislação e criação de um programa de prevenção e controle de infecção hospitalar (Infecção relacionada à assistência à saúde - IRAS). Disponível em: http://www.anvisa.gov. $\mathrm{br} /$ servicosaude/manuais/iras/M\%F3dulo\%201\%20-\%20Legisla\%E7\%E3o\%20e\%20Programa\%20de\%20 Preven\%E7\%E3o\%20e\%20Controle\%20de\%20Infec\%E7\%E3o\%20Hospitalar.pdf. Acesso em: 13 fev. 2015.

BRANDALISE, L. T.; PERTILE, A.; BORTOLI, D. (2009). Gestão hospitalar e a logística: um estudo de caso. Ciências Sociais em Perspectiva, v.8, n.13, 2009.

CAMPOS, C. J. G. (2004). Método de análise de conteúdo: ferramenta para a análise de dados qualitativos no campo da saúde. Revista Brasileira de Enfermagem, v. 57, n.5, p. 611-614, 2004.

CAMPOS, J. F.; DAVID, H. S. L. Avaliação do contexto de trabalho em terapia intensiva sob o olhar da psicodinâmica do trabalho. Revista da Escola de Enfermagem da USP, v. 45, n. 2, p. 363-368, 2011.

CODO, W. Um diagnóstico integrado do trabalho com ênfase em saúde mental. In: JACQUES, M. G.,; CODO, W. (Orgs.). Saúde mental e trabalho. Petrópolis, RJ: Vozes, 2002.

CONSELHO NACIONAL DE SAÚDE. Resolução CNS $\mathbf{n}^{\circ} 466$ de 12 de dezembro de 2012. Aprova as diretrizes e normas regulamentadoras de pesquisas envolvendo seres humanos. Disponível em: http://conselho. saude.gov.br/resolucoes/2012/Reso466.pdf. Acesso em: 24 fev. 2015.

FIGUEIREDO, M. L.; ZUFFI, F. B. Cuidados aos portadores de úlcera venosa: percepção dos enfermeiros da Estratégia de Saúde da Família. Enfermería Global, v. 28, p. 147-158, 2012.

FREITAS, W. R. S.; C. J. C., JABBOUR (2011). Utilizando estudo de caso(s) como estratégia de pesquisa qualitativa: boas práticas e sugestões. Estudo \& Debate, v. 18, n. 2, p. 07-22, 2011.

GARCIA, S. D.; GIL, R. B.; LAUS, A. M.; HADDAD, M. C. L.; VANNUCHI M. T. O.; TALDIVO, M. A. Gerenciamento de recursos materiais na prática da higienização das mãos. Revista de Enfermagem da UFPE on-line, v. 7, n. 5, p. 1342-1348, 2013.

GARCIA, S. D.; HADDAD, M. C. L.; DELlAROZA, M. S. G; COSTA, D. B.; MIRANDA, J. M. Gestão de material médico-hospitalar e o processo de trabalho em um hospital público. Revista Brasileira de Enfermagem, v. 65, n. 2, p. 339-346, 2012.

GODOY, A. S. Introdução à pesquisa qualitativa e suas possibilidades. Revista de Administração de Empresas, v. 35, n. 2, p. 57-63, 1995.

GOMES, A. M. Enfermagem na unidade de terapia intensiva. São Paulo, SP: EPU, 1998. 
GRITTEM, L.; MEIER, M. J.; ZAGONEL, I. P. S. Pesquisa-ação: uma alternativa metodológica para pesquisa em enfermagem. Texto and Contexto Enfermagem, v. 17, n. 4, p. 765, 2008.

HOCHMAN, B; NAHAS, F. X.; OLIVEIRA FILHO, R.S.; FERREIRA, L. M. Desenhos de pesquisa. Acta Cirúrgica Brasileira, v. 20, Supl. 2, p. 1-9, 2005.

HONÓRIO, M. T.; ALBUQUeRQUE, G. L. A gestão de materiais em enfermagem. Ciência, Cuidado e Saúde, v. 4, n. 3, p. 259-268, 2008.

KOIZUMI, M. S.; KAMIYAMA, Y.; FREITAS, L. A. Percepção dos pacientes de unidade de terapia intensiva problemas sentidos expectativas em relação à assistência de enfermagem. Revista da Escola de Enfermagem USP, v. 13, n. 2, p. 135-145, 1979.

KOVÁCS, M. J. (2010). Sofrimento da equipe de saúde no contexto hospitalar: cuidando do cuidador profissional. O Mundo da Saúde, v. 34, n. 4, p. 420-429, 2010.

LANGE, L. M. J.; FIORELLI, R. A. L P. Vivências emocionais de equipes de saúde trabalhando em UTI pediátrica: Um olhar transpessoal sobre o sofrimento e o morrer. (Monografia). Graduação em Psicologia Transpessoal, ALUBRAT - CESBLU, Campinas, 2009.

LANNA, E. (2010). A importância da gestão eficiente de recursos materiais na área hospitalar. Recuperado em 14 junho, 2014, de http://www.administradores.com.br/artigos/economia-e-financas/a-importancia-dagestao-eficiente-de-recursos-materiais-na-area-hospitalar/50386/. Acesso em: 19 mar. 2015.

LEITE, M. A.; VILA, V. S. C. Dificuldades vivenciadas pela equipe multiprofissional na unidade de terapia intensiva. Revista Latino-americana de Enfermagem, v. 13, n. 2, p. 145-150, 2005.

MADUREIRA, C. R.; VEIGA, K.; SANT'ANA, A. F. M. Gerenciamento de tecnologia em terapia intensiva. Revista Latinoamericana de Enfermagem, v. 8, n. 6, p. 68-75, 2000.

MANZINI, E. J. (2003). Uso da Entrevista em Dissertações e Teses Produzidas em um Programa de PósGraduação em Educação. Disponível em: http://pt.scribd.com/doc/244400669/Artigo-Uso-da-entrevistaEduardo-Manzini-pdf\#scribd. Acesso em: 19 nov. 2014.

MENDES, K. G. L.; CASTILHO, V. Determinação da importância operacional dos materiais de enfermagem segundo a Classificação XYZ. Revista do Instituto de Ciências da Saúde, v. 27, n. 4, p. 324-329, 2009.

MESQUITA, S. R. A. M., ANSELMI, M. L., SANTOS, C. B., HAYASHIDA, M. Programa interdisciplinar de interação domiciliar de Marília-SP: custos de recursos materiais consumidos. Revista Latinoamericana de Enfermagem, v. 13, n. 4, p. 555-561, 2005.

MINISTÉRIO DA SAÚDE. Portaria GM no 2616 de 12 de maio de 1998. Discorre sobre o Programa de Controle de Infecções Hospitalares (PCIH). Disponível em: http://portal.anvisa.gov.br/wps/wcm/connec t/8c6cac8047457a6886d6d63fbc4c6735/PORTARIA+N\%C2\%B0+2.616,+DE+12+DE+MAIO+DE+1998. pdf?MOD=AJPERES. Acesso em: 19 mar. 2015.

OLIVEIRA, A. D.; COSTA, C. R.; ARNDT, A. B. M. Glosas de materiais e medicamentos em um hospital privado na cidade de Brasília, Distrito Federal. Acta de Ciências e Saúde, v. 1, n.2, p. 1-12, 2012.

OLIVEIRA, N. C.; CHAVES, L. D. P. Gerenciamento de recursos materiais: o papel da enfermeira de unidade de terapia intensiva. Revista da Rede de Enfermagem de Fortaleza, v. 10, n. 4, p. 19-23, 2009. 
PADILHA, K. G.; KIMURA, M. Aspectos éticos da prática de enfermagem em Unidade de Terapia Intensiva. Sobeti em Revista, v. 1, n. 1, p. 8-11, 2000.

PASCHOAL, M. L. H.; CASTILHO, V. C. Consumo de materiais em centro cirúrgico após implementação de sistema de gestão informatizado. Revista Brasileira de Enfermagem, v. 63, n. 6, p. 887-893, 2010.

PAULUS, A. Jr. Gerenciamento de recursos materiais em unidades de saúde. Revista Espaço para a Saúde, v. 7, n. 1, p. 30-45, 2005.

PEREIRA, F. M. V.; MALAGUTI-TOFFANO, S. E.; SILVA, A. M.; CANINI, S. R. M. S.; GIR, E. Adesão às precauções-padrão por profissionais de enfermagem que atuam em terapia intensiva em um hospital universitário. Revista da Escola de Enfermagem da USP, v. 47, n. 3, p. 686-693, 2013.

PIOVESAN, A.; TEMPORINI, E. R. Pesquisa exploratória: procedimento metodológico para o estudo de fatores humanos no campo da saúde pública. Revista de Saúde Pública, v. 29, n. 4, p. 318-325, 1995.

RIBEIRO, E. M.; PIRES, D.; BLANK, V. L. G. A teorização sobre processo de trabalho em saúde como instrumental para análise do trabalho no Programa Saúde da Família. Cadernos de Saúde Pública, v. 20, n. 2, p. 438-446, 2004.

ROBERTO, W. L. C.; LIRA, R. A. O gestor hospitalar e sua atuação frente ao suprimento de materiais. Perspectiva On-line, v. 4, n. 13, p. 87-104, 2010.

SANTOS, K. M. A. B.; SILVA, M. J. P. Percepção dos profissionais de saúde sobre a comunicação com os familiares de pacientes em UTIs. Revista Brasileira de Enfermagem, v. 59, n. 1, p. 61-66, 2006.

SUSAKI,T. T.; SILVA, M. J. P.; POSSARI, J. F. Identificação das fases do processo de morrer pelos profissionais de Enfermagem. Acta Paulista de Enfermagem, v. 19, n. 2, p. 144-149, 2006.

TRIVIÑOS, A. N. S. Introdução à pesquisa em ciências sociais: a pesquisa qualitativa em educação. São Paulo: Atlas, 1987.

VAGHETTI, H. H.; ROEHRS, M.; PIRES, A. C.; RODRIGUEZ, C. Desperdício de materiais assistenciais na percepção de trabalhadores de enfermagem de um hospital universitário. Revista de Enfermagem da UERJ, v. 19, n. 3, p. 369-374, 2011.

VECINA NETO, G.; REINHARDT FILHO, W. Gestão de recursos materiais e de medicamentos, Série Saúde \& Cidadania, v. 12. São Paulo: Faculdade de Saúde Pública da Universidade de São Paulo, 1998.

VOSS, C.; TSIKRIKTSIS, N.; FROHLICH, M. Case research in operations management. International Journal of Operations \& Production Management, v. 22, n. 2, p. 195-219, 2002. 